EPiC Series in Computing
Volume 80, 2021, Pages 176-183
8th International Workshop on Applied Verifica-
tion of Continuous and Hybrid Systems (ARCH21)

\title{
ORBITADOR: A tool to analyze the stability of periodical dynamical systems
}

\author{
Jawher Jerray ${ }^{1}$ \\ Université Sorbonne Paris Nord, LIPN, CNRS, F-93430 Villetaneuse, France \\ jerrayalipn.univ-paris13.fr
}

\begin{abstract}
Tool Presentation: We present ORBITADOR, a tool for stability analysis of dynamical systems. ORBITADOR uses a method that generates a bounded invariant set of a differential system with a given set of initial conditions around a point $x_{0}$ to prove the existence of a limit cycle. This invariant has the form of a tube centered on the Euler approximate solution starting at $x_{0}$, which has for radius an upper bound on the distance between the approximate solution and the exact ones. The method consists in finding a real $T>0$ such that the "snapshot" of the tube at time $t=(i+1) T$ is included in the snapshot at $t=i T$, for some integer $i$ with adding a small bounded uncertainty. This uncertainty allows using an approximate value $T$ of the exact period. We successfully applied ORBITADOR to several classical examples of periodical systems.
\end{abstract}

\section{Introduction}

The analysis of stability and convergence of numerical schemes for differential systems is often based on the property of contraction of the space of solutions [2,11].

Given a differential system $\Sigma: d x / d t=f(x)$ of dimension $n$, an initial point $x_{0} \in \mathbb{R}^{n}$, a real $\varepsilon>0$, and a ball $B_{0}=B\left(x_{0}, \varepsilon\right)^{1}$, we present the tool which implements a method allowing to find a bounded invariant set of $\Sigma$ containing the trajectories starting at $B_{0}$. We first add a (small) bounded uncertainty $w(t)$ to the system, whose values belong to a convex set $\mathcal{W}$ of dimension $m$. We thus obtain a disturbed system of the form $\Sigma_{\mathcal{W}}: d x / d t=f(x, w)$, and we look for a bounded (forward) invariant set for $\Sigma_{\mathcal{W}}$. This invariant set has the form of a tube whose center at time $t$ is the Euler approximate solution $\tilde{x}(t)$ of the system starting at $x_{0}$, and radius is a function $\delta_{\varepsilon, \mathcal{W}}(t)$ bounding the distance between $\tilde{x}(t)$ and any exact solution $x(t)$ of $\Sigma_{\mathcal{W}}$ starting at $B_{0}$. The tube can thus be described as $\bigcup_{t \geq 0} B_{\mathcal{W}}(t)$ where $B_{\mathcal{W}}(t) \equiv B\left(\tilde{x}(t), \delta_{\varepsilon, \mathcal{W}}(t)\right)$. To find a bounded invariant set, we then look for a positive real $T$ such that $B_{\mathcal{W}}((i+1) T) \subseteq B_{\mathcal{W}}(i T)$ for some $i \in \mathbb{N}$. In case of success, we show that the set $I_{\mathcal{W}}=\bigcup_{t \in[i T,(i+1) T]} B_{\mathcal{W}}(t)$ constitutes a bounded invariant set for $\Sigma_{\mathcal{W}}$ (and also for $\Sigma$ corresponding to the particular case $\mathcal{W}=0$ ).

The integrated approach in ORBITADOR allows to study the stability of dynamical systems.

\footnotetext{
${ }^{1} B\left(x_{0}, \varepsilon\right)$ is the set $\left\{z \in \mathbb{R}^{n} \mid\left\|z-x_{0}\right\| \leq \varepsilon\right\}$ where $\|\cdot\|$ denotes the Euclidean distance.
} 
Outline: Section 2, describes the method used in ORBITADOR to proof the existing of limit cycles and study the stability of differential systems. In Section 3, we expose the organization and the features of ORBITADOR. Section 4 gives the results obtained on the biped example. Section 5 concludes this work.

\section{Method}

\subsection{Explicit Euler time integration and error bounds}

Let us consider the differential system:

$$
\frac{d x(t)}{d t}=f(x(t))
$$

with states $x(t) \in \mathbb{R}^{n}$. denotes the state of the system at time $t$.

We will use $x\left(t ; x_{0}\right)$ (or sometimes just $\left.x(t)\right)$ to denote the exact continuous solution of the system at time $t$, for a given initial condition $x_{0}$.

We use $\tilde{x}\left(t ; y_{0}\right)$ (or just $\left.\tilde{x}(t)\right)$ to denote Euler's approximate value of $x\left(t ; y_{0}\right)$ (defined by $\tilde{x}\left(t ; y_{0}\right)=$ $y_{0}+t f\left(y_{0}\right)$ for $t \in[0, \tau]$, where $\tau$ is the integration time-step).

We suppose that we know a bounded region $\mathcal{S} \subset \mathbb{R}^{n}$ containing the solutions of the system for a set of initial conditions $B_{0}$ and a certain amount of time.

We now give an upper bound to the error between the exact solution of the ODE and its Euler approximation on $\mathcal{S}$ (see [10,9]).

Definition 1. Let $\varepsilon$ be a given positive constant. Let us define, for $t \in[0, \tau], \delta_{\varepsilon}(t)$ as follows:

$$
\begin{aligned}
& \text { if } \lambda<0: \delta_{\varepsilon}(t)=\left(\varepsilon^{2} e^{\lambda t}+\frac{C^{2}}{\lambda^{2}}\left(t^{2}+\frac{2 t}{\lambda}+\frac{2}{\lambda^{2}}\left(1-e^{\lambda t}\right)\right)\right)^{\frac{1}{2}} \\
& \text { if } \lambda=0: \delta_{\varepsilon}(t)=\left(\varepsilon^{2} e^{t}+C^{2}\left(-t^{2}-2 t+2\left(e^{t}-1\right)\right)\right)^{\frac{1}{2}} \\
& \text { if } \lambda>0: \delta_{\varepsilon}(t)=\left(\varepsilon^{2} e^{3 \lambda t}+\frac{C^{2}}{3 \lambda^{2}}\left(-t^{2}-\frac{2 t}{3 \lambda}+\frac{2}{9 \lambda^{2}}\left(e^{3 \lambda t}-1\right)\right)\right)^{\frac{1}{2}}
\end{aligned}
$$

where $C$ and $\lambda$ are real constants specific to function $f$, defined as follows:

$$
C=\sup _{y \in \mathcal{S}} L\|f(y)\|
$$

where $L$ denotes the Lipschitz constant for $f$, and $\lambda$ is the "one-sided Lipschitz constant" (or "logarithmic Lipschitz constant" [2]) associated to $f$, i. e., the minimal constant such that, for all $y_{1}, y_{2} \in \mathcal{S}$ :

$$
\left\langle f\left(y_{1}\right)-f\left(y_{2}\right), y_{1}-y_{2}\right\rangle \leq \lambda\left\|y_{1}-y_{2}\right\|^{2}
$$

where $\langle\cdot, \cdot\rangle$ denotes the scalar product of two vectors of $\mathcal{S}$.

The constant $\lambda$ can be computed using a nonlinear optimization solver (e.g., Sequential quadratic programming (SQP) [7]) or using the Jacobian matrix of $f$ (see, e. g., [2]).

Proposition 1. [9] Consider the solution $x\left(t ; y_{0}\right)$ of $\frac{d x}{d t}=f(x)$ with initial condition $y_{0}$ and the approximate Euler solution $\tilde{x}\left(t ; x_{0}\right)$ with initial condition $x_{0}$. For all $y_{0} \in B\left(x_{0}, \varepsilon\right)$, we have:

$$
\left\|x\left(t ; y_{0}\right)-\tilde{x}\left(t ; x_{0}\right)\right\| \leq \delta_{\varepsilon}(t)
$$




\subsection{Systems with bounded uncertainty}

Let us now show how the method extends to systems with "bounded uncertainty". A differential system with bounded uncertainty is of the form

$$
\frac{d x(t)}{d t}=f(x(t), w(t))
$$

with $t \in \mathbb{R}_{>0}^{n}$, states $x(t) \in \mathbb{R}^{n}$, and uncertainty $w(t) \in \mathcal{W} \subset \mathbb{R}^{m}(\mathcal{W}$ is compact, i. e., closed and bounded). We assume that any bounded uncertainty trajectory is always bounded in the compact set $\mathcal{W}$. We denote this by $w(\cdot) \in \mathcal{W}$, i. e., $w(t) \in \mathcal{W}, \forall t \geq 0$ (see [14, 13]). We suppose (see [8]) that there exist constants $\lambda \in \mathbb{R}$ and $\gamma \in \mathbb{R}_{\geq 0}$ such that, for all $y_{1}, y_{2} \in \mathcal{S}$ and $w_{1}, w_{2} \in \mathcal{W}$ :

$$
\left\langle f\left(y_{1}, w_{1}\right)-f\left(y_{2}, w_{2}\right), y_{1}-y_{2}\right\rangle \leq \lambda\left\|y_{1}-y_{2}\right\|^{2}+\gamma\left\|y_{1}-y_{2}\right\|\left\|w_{1}-w_{2}\right\| \quad(H 1) .
$$

This formula can be seen as a generalization of (H0) (see Section 2.1). Recall that $\lambda$ has to be computed in the absence of uncertainty $(\mathcal{W}=0)$. The additional constant $\gamma$ is used for taking into account the uncertainty $w$. Instead of computing them globally for $\mathcal{S}$, it is advantageous to compute $\lambda$ and $\gamma$ locally depending on the subregion of $\mathcal{S}$ occupied by the system state during a considered interval of time. We now give a version of Proposition 1 with bounded uncertainty $w(\cdot) \in \mathcal{W}$, originally proved in [8].

Proposition 2. [8] Consider a system $\Sigma_{\mathcal{W}}$ with bounded uncertainty of the form $\frac{d x(t)}{d t}=f(x(t), w(t))$ satisfying (H1). Consider a point $x_{0} \in \mathcal{S}$ and a point $y_{0} \in B\left(x_{0}, \varepsilon\right)$. Let $x\left(t ; y_{0}\right)$ be the exact solution of the system $\Sigma_{\mathcal{W}}$, initial condition $y_{0}$, and $\tilde{x}\left(t ; x_{0}\right)$ the Euler approximate solution of the system $\frac{d x(t)}{d t}=$ $f(x(t), 0)$ without uncertainty $(\mathcal{W}=0)$ with initial condition $x_{0}$. We have, for all $w(\cdot) \in \mathcal{W}$ and $t \in[0, \tau]:$

$$
\left\|x\left(t ; y_{0}\right)-\tilde{x}\left(t ; x_{0}\right)\right\| \leq \delta_{\varepsilon, \mathcal{W}}(t)
$$

with

$$
\begin{aligned}
& \text { if } \lambda<0: \delta_{\varepsilon, \mathcal{W}}(t)=\left(\frac{C^{2}}{-\lambda^{4}}\left(-\lambda^{2} t^{2}-2 \lambda t+2 e^{\lambda t}-2\right)+\frac{1}{\lambda^{2}}\left(\frac{C \gamma|\mathcal{W}|}{-\lambda}\left(-\lambda t+e^{\lambda t}-1\right)\right.\right. \\
&\left.\left.+\lambda\left(\frac{\gamma^{2}(|\mathcal{W}| / 2)^{2}}{-\lambda}\left(e^{\lambda t}-1\right)+\lambda \varepsilon^{2} e^{\lambda t}\right)\right)\right)^{1 / 2} \\
& \text { if } \lambda>0: \delta_{\varepsilon, \mathcal{W}}(t)=\frac{1}{(3 \lambda)^{3 / 2}}\left(\frac{C^{2}}{\lambda}\left(-9 \lambda^{2} t^{2}-6 \lambda t+2 e^{3 \lambda t}-2\right)+3 \lambda\left(\frac{C \gamma|\mathcal{W}|}{\lambda}\left(-3 \lambda t+e^{3 \lambda t}-1\right)\right.\right. \\
&+\left.\left.3 \lambda\left(\frac{\gamma^{2}(|\mathcal{W}| / 2)^{2}}{\lambda}\left(e^{3 \lambda t}-1\right)+3 \lambda \varepsilon^{2} e^{3 \lambda t}\right)\right)\right)^{1 / 2} \\
& \text { if } \lambda=0: \delta_{\varepsilon, \mathcal{W}}(t)=\left(C^{2}\left(-t^{2}-2 t+2 e^{t}-2\right)+\left(C \gamma|\mathcal{W}|\left(-t+e^{t}-1\right)\right.\right. \\
&+\left.\left.\left(\gamma^{2}(|\mathcal{W}| / 2)^{2}\left(e^{t}-1\right)+\varepsilon^{2} e^{t}\right)\right)\right)^{1 / 2}
\end{aligned}
$$

where $|\mathcal{W}|$ is the diameter of $\mathcal{W}$ (maximum distance between 2 points of $\mathcal{W}$ ).

\subsection{Correctness}

Consider a differential system $\Sigma_{\mathcal{W}}: d x / d t=f(x, w)$ with $w \in \mathcal{W}$, an initial point $x_{0} \in \mathbb{R}^{n}$, a real $\varepsilon>$ 0 and a ball $B_{0}=B_{\mathcal{W}}\left(x_{0}, \varepsilon\right)$. Let $B_{\mathcal{W}}(t)$ denote $B\left(\tilde{x}(t), \delta_{\varepsilon, \mathcal{W}}(t)\right)$ where $\tilde{x}(t)$ is the Euler approximate solution of the system without uncertainty and initial condition $x_{0}{ }^{2}$. It follows from Proposition 2

\footnotetext{
${ }^{2}$ Note that $B_{\mathcal{W}}(0)=B_{0}$ because $\tilde{x}(0)=x_{0}$ and $\delta_{\varepsilon, \mathcal{W}}(0)=\varepsilon$.
} 
that $\bigcup_{t>0} B_{\mathcal{W}}(t)$ is an invariant set containing $B_{0}$. If moreover, we can find an integer $i \geq 0$ and a real $T=k \tau$ (for some $k \in \mathbb{N}$ ) such that $B_{\mathcal{W}}((i+1) T) \subseteq B_{\mathcal{W}}(i T)$, then the set $I_{\mathcal{W}}=\bigcup_{t \in[i T,(i+1) T]} B_{\mathcal{W}}(t)$ is a bounded invariant which contains all the solutions $x(t)$ of $\Sigma_{\mathcal{W}}$ starting at $B_{0}$, for $t \in[i T, \infty)$. In the phase space, this bounded invariant has a "torus" shape. We have (see [4]):

Proposition 3. Suppose that there exist $T>0$ (with $T=k \tau$ for some $k$ ) and $i \in \mathbb{N}$ such that: $B_{\mathcal{W}}((i+1) T) \subseteq B_{\mathcal{W}}(i T)$. Then $I_{\mathcal{W}} \equiv \bigcup_{t \in[i T,(i+1) T]} B_{\mathcal{W}}(t)$ is a compact (i.e., bounded and closed) invariant set containing, for $t \in[i T, \infty)$, all the solutions of $\Sigma_{\mathcal{W}}$ with initial condition in $B_{0}$. The set $I_{\mathcal{W}}$ is also an invariant for the unperturbed system $\Sigma$.

Proof. All solutions $x(t)$ of $\Sigma_{\mathcal{W}}$ starting from $B_{0}$ are contained for $t=(i+1) T$ in $B_{\mathcal{W}}((i+1) T)$ by Proposition 2, hence in $B_{\mathcal{W}}(i T)$ by inclusion hypothesis. A solution starting from $B_{\mathcal{W}}((i+1) T) \subseteq$ $B_{\mathcal{W}}(i T)$ after an additional time $t^{\prime}=T$ finds itself in in $B_{\mathcal{W}}((i+1) T) \subseteq B_{\mathcal{W}}(i T)$. And so on, any solution of $I_{\mathcal{W}}$ starting from $B_{\mathcal{W}}(i T)$ returns periodically (with period $T$ ) in $B_{\mathcal{W}}(i T)$. Similarly, any point of $B_{\mathcal{W}}\left(i T+t^{\prime}\right)$ with $t^{\prime} \in[0, T)$ returns periodically (with period $T$ ) in $B_{\mathcal{W}}\left(i T+t^{\prime}\right)$. The set $I_{\mathcal{W}}=\bigcup_{t \in[i T,(i+1) T]} B_{\mathcal{W}}(t)$ is thus an invariant of $\Sigma_{\mathcal{W}}$. It is also an invariant set for the unperturbed system $\Sigma$ corresponding to the particular case $\mathcal{W}=0$.

Theorem 1. Suppose that there exist $T>0$ (with $T=k \tau$ for some $k$ ) and $i \in \mathbb{N}$ such that: $B_{\mathcal{W}}((i+$ 1) $T) \subseteq B_{\mathcal{W}}(i T)$. Then there exists a closed orbit (limit cycle or fixed-point) for the unperturbed system $\Sigma$ which is contained in $I_{\mathcal{W}}$.

Proof. (Sketch) Consider a section $V$ tranverse to the flow of the unperturbed system $\Sigma$, and the "first return map" (or Poincaré map) $\mathcal{T}$ from $V \cap I_{\mathcal{W}}$ to $V$. This mapping is known to be continuous (and even differentiable). Besides $\mathcal{T}\left(V \cap I_{\mathcal{W}}\right) \subseteq V \cap I_{\mathcal{W}}$ because $I_{\mathcal{W}}$ is an invariant set of $\Sigma$. Therefore, by Brouwer's fixed-point theorem, there is a point $M \in V \cap I_{\mathcal{W}}$ such that $\mathcal{T}(M)=M$. It follows that the solution of $\Sigma$ starting at $M$ returns to $M$ after some time, say $T^{*}$. This defines a closed periodic orbit of $\Sigma$ passing by $M$ of period $T^{*}$.

\section{ORBITADOR organization and principle}

ORBITADOR is a tool that implements a formal method to prove formally the stability of dynamical systems governed by differential equations.

\subsection{Targeted user}

The tool is intended to the user wishing to verify the existence of an attractive limit cycle, and to construct an invariant set enclosing it.

\subsection{Global architecture}

ORBITADOR is written in Python, it is made of 1,037 lines of code, and can therefore run under any operating system. We explain in Section 3.2 the global architecture of the system, that starts by the input Python file describing the ODE and all specifications of the computed system. Using this input, the method defined in Section 2 generates an invariant set $I_{\mathcal{W}}$ that allows study the stability of the given system. Graphical an numerical results can be provided as the output of the analysis.

The input file of ORBITADOR is composed of 3 main modules: 


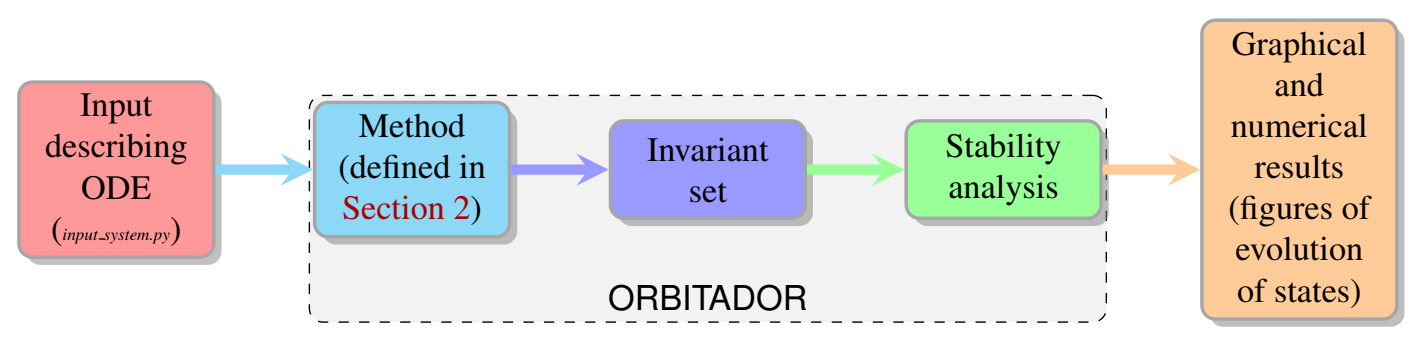

Figure 1: Workflow of ORBITADOR

1. Problem Definition: In this module, the user defines the names of the states that constitute the system, also the user specifies the names of the parameters and their values. After that, the user gives the differential equations of the system. If the problem contains a guard condition and a reset, it is possible to add them in the problem definition part.

2. Problem Configuration: In the problem configuration module, the user can set the options to run ORBITADOR. Among these options, we find the time steps, the initial conditions of the system or the starting point at initial time $t_{0}$ the value of the uncertainty $\mathcal{W}$ and the number of the periods.

3. Visualization: In the last module, the user specify the states to display. It can be a 1D plot where the state is shown as a function of $t$ or a phase portal where a state is shown as a function of an other state, in this case it can be 2D or 3D plot (see, e. g., Fig. 3 for an example of a 2D-plot).

To launch ORBITADOR the user needs to run, in the terminal, the executable file orbitador followed by the name of the input file:

- /orbitador input_system.py

As shown in Fig. 2, to analyze a dynamical system using ORBITADOR, the user needs to give as inputs its differential equations and initial conditions, also he needs to provide the time step $\tau$, the number of periods $N$ in order to fix a maximum duration $T_{f}=N T$ in which ORBITADOR tries to find the index sought $i$ (as defined in Proposition 3), the bounded uncertainty $\mathcal{W}$ and the radius $\varepsilon$ of the initial ball $B_{0}$. An approximate value $T(=k \tau$ for some integer $k$ ) of the period of the system can be computed automatically by ORBITADOR or it can be fixed by the user as an input.

Using those inputs, ORBITADOR computes the local values of $\lambda, L, C$ and $\gamma$ as well as the function $\delta_{\varepsilon, W}(\cdot)$ (as defined in Definition 1 and Section 2.2). Then ORBITADOR outputs the invariant set $I_{\mathcal{W}}$ (as defined in Proposition 3).

The tool, its source code, several examples and results are available on the website of ORBITADOR https://lipn.univ-paris13.fr/ jerray/orbitador/.

\section{Example: Passive biped model}

It is possible to use ORBITADOR in order to analyze the stability of hybrid systems, i. e., continuous systems which, upon the satisfaction of a certain state condition ("guard"), may reset instantaneously the state before resuming the application of ODEs. Many works in the domain of symbolic control have explained how to compute an overapproximation of the intersection of the current set of reachability with the guard condition, and perform the reset operation (see, e. g., [3, 1, 6]). We describe here the results of such an extension to the passive biped model [12], seen as a hybrid oscillator. The passive biped model exhibits indeed a stable limit-cycle oscillation for appropriate parameter 


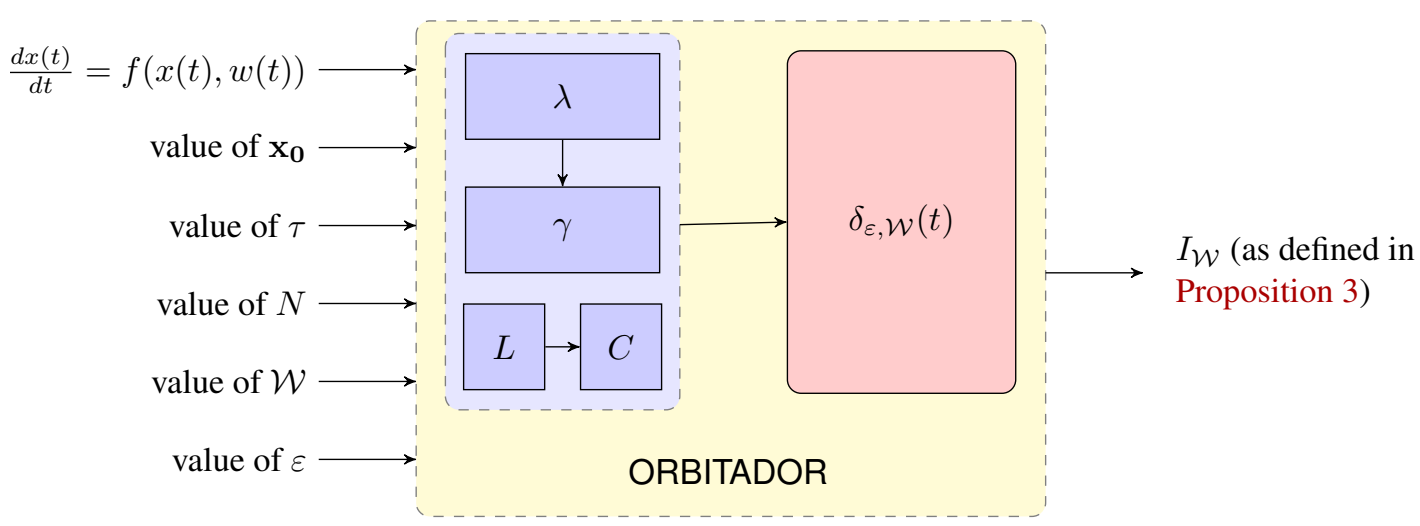

Figure 2: ORBITADOR's structure

values that corresponds to periodic movements of the legs [15]. The model has a continuous state variable $\boldsymbol{x}(t)=\left(\phi_{1}(t), \dot{\phi}_{1}(t), \phi_{2}(t), \dot{\phi}_{2}(t)\right)^{\top}$. The dynamics is described by $\frac{\mathbf{d} \boldsymbol{x}(\mathbf{t})}{\mathbf{d t}}=\boldsymbol{f}(\boldsymbol{x})+w$ with $w \in \mathcal{W} \subset \mathbb{R}^{4}$ and

$$
\begin{aligned}
& \boldsymbol{f}(\boldsymbol{x})=\left(\begin{array}{c}
\dot{\phi}_{1} \\
\sin \left(\phi_{1}-\gamma\right) \\
\dot{\phi}_{2} \\
\dot{\sin }\left(\phi_{1}-\gamma\right)+\dot{\phi}_{1}^{2} \sin \phi_{2}-\cos \left(\phi_{1}-\gamma\right) \sin \phi_{2}
\end{array}\right), \operatorname{Reset}(\boldsymbol{x})=\left(\begin{array}{c}
-\phi_{1} \\
\dot{\phi}_{1} \sin \left(2 \phi_{1}\right) \\
-2 \phi_{1} \\
\dot{\phi_{1}} \cos 2 \phi_{1}\left(1-\cos 2 \phi_{1}\right)
\end{array}\right) \\
& \operatorname{Guard}(\boldsymbol{x}) \equiv\left(2 \phi_{1}-\phi_{2}=0 \wedge \phi_{2}<-\delta\right) .
\end{aligned}
$$

The time-step used in Euler's method is $\tau=2 \cdot 10^{-5}$, and ORBITADOR then automatically computes an approximate value of the period equal to $T=k \tau$ with $k=194129$. Also, we choose $N=5$ as the number of periods. Therefore, the maximum duration $T_{f}$ of this experience is $T_{f}=N T=5 T=19.4129 s$. For a system with $\mathcal{W}=[-0.0001,0.0001]^{4}$ and set of initial conditions $B\left(x_{0}, \varepsilon\right)$ with $\varepsilon=0.01$ and $x_{0}=(0.009,-0.05869,-0.0009629,-0.3432)$, ORBITADOR finds: $B_{\mathcal{W}}\left(\left(i_{0}+1\right) T\right) \subset B_{\mathcal{W}}\left(i_{0} T\right)$ for $i_{0}=4$.

On Fig. 3, the curve of Euler's approximation $\tilde{x}(t)$ is depicted in red for initial condition $x_{0}$ and $t \in\left[0, T_{f}\right]$. The borders of the tube $B_{\mathcal{W}}(t) \equiv B\left(\tilde{x}(t), \delta_{\varepsilon, \mathcal{W}}(t)\right)$ are depicted in green. The black vertical lines delimit the portion of the tube between $t=i_{0} T$ and $t=\left(i_{0}+1\right) T$ corresponding to the invariant set $I_{\mathcal{W}}$ enclosing the limit cycle.

It follows by Theorem 1 that, for any initial condition in $B\left(x_{0}, \varepsilon\right)$, the system converges towards an attractive limit cycle contained in $I_{\mathcal{W}} \equiv \bigcup_{t \in[4 T, 5 T]} B_{\mathcal{W}}(t)$.

The experiment above is performed on an ASUS X411UN Intel Core i7-8550U $1.80 \mathrm{GHz}$ with $8 \mathrm{GiB}$ memory running Linux Mint 19. The proof takes 327 seconds of CPU time.

\section{Conclusion}

We presented ORBITADOR, a tool coded in Python that guarantees the existence of limit cycles and constructs invariant sets around them. ORBITADOR uses a very general criterion of inclusion of one set 

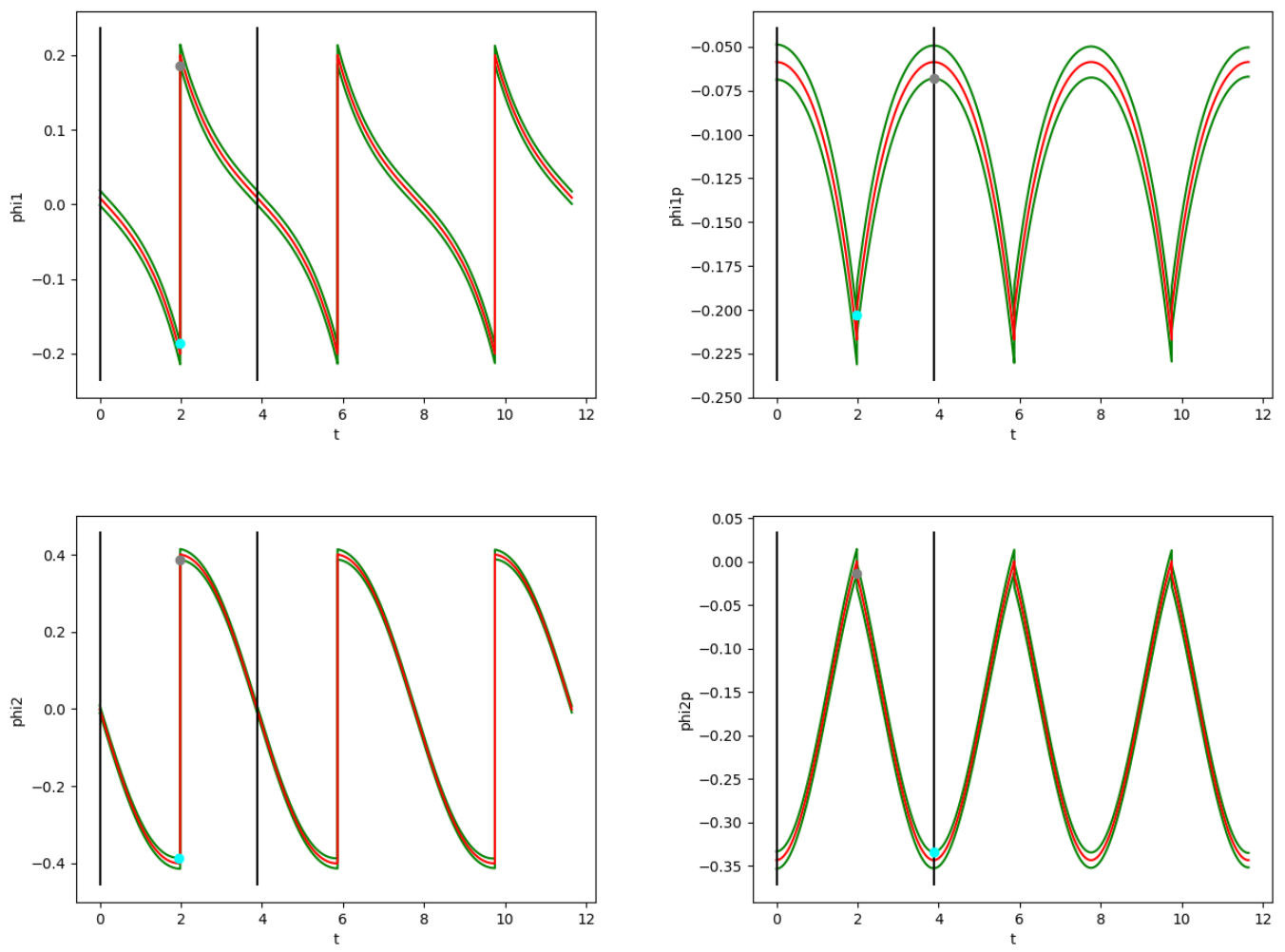

Figure 3: Biped system with uncertainty $w \in \mathcal{W}=[-0.0001,0.0001]^{4}$, initial radius $\varepsilon=0.001$, approximate period $T=3.8826 \mathrm{~s}$ and time-step $\tau=2 \cdot 10^{-5}$.

in another. This is simpler than classical criteria based on contractivity properties or Lyapunov functions. Also, by adding a small bounded uncertainty $w \in \mathcal{W}$ to the system, we can use an approximate value $T$ of the period and not an exact value.

In future work, we plan to upgrade ORBITADOR so that it computes dynamical systems with control (see [5]).

\section{References}

[1] Matthias Althoff and Bruce H. Krogh. Avoiding geometric intersection operations in reachability analysis of hybrid systems. In HSCC, pages 45-54. ACM, 2012.

[2] Zahra Aminzare and Eduardo D. Sontag. Contraction methods for nonlinear systems: A brief introduction and some open problems. In 53rd IEEE Conference on Decision and Control, CDC 2014, Los Angeles, CA, USA, December 15-17, 2014, pages 3835-3847, 2014.

[3] Antoine Girard and Colas Le Guernic. Zonotope/hyperplane intersection for hybrid systems reachability analysis. In HSCC, volume 4981 of $L N C S$, pages 215-228. Springer, 2008.

[4] Jawher Jerray and Laurent Fribourg. Determination of limit cycles using stroboscopic set-valued maps. In 7 th IFAC Conference on Analysis and Design of Hybrid Systems (ADHS'21), July 7-9, 2021, Brussels, Belgium, 2021. 
[5] Jawher Jerray, Laurent Fribourg, and Étienne André. Robust optimal periodic control using guaranteed euler's method. In ACC, 2021. To appear.

[6] Niklas Kochdumper and Matthias Althoff. Reachability analysis for hybrid systems with nonlinear guard sets. In $H S C C$, pages 1:1-1:10, 2020.

[7] Dieter Kraft et al. A software package for sequential quadratic programming. 1988.

[8] Adrien Le Coënt, Julien Alexandre Dit Sandretto, Alexandre Chapoutot, Laurent Fribourg, Florian De Vuyst, and Ludovic Chamoin. Distributed control synthesis using Euler's method. In Proc. of International Workshop on Reachability Problems (RP'17), volume 247 of Lecture Notes in Computer Science, pages 118-131. Springer, 2017.

[9] Adrien Le Coënt, Florian De Vuyst, Ludovic Chamoin, and Laurent Fribourg. Control synthesis of nonlinear sampled switched systems using Euler's method. In Proc. of International Workshop on Symbolic and Numerical Methods for Reachability Analysis (SNR'17), volume 247 of EPTCS, pages 18-33. Open Publishing Association, 2017.

[10] Adrien Le Coënt and Laurent Fribourg. Guaranteed optimal reachability control of reaction-diffusion equations using one-sided Lipschitz constants and model reduction. In Model-Based Design of Cyber Physical Systems (CyPhy'19), N.-Y., USA, 2019.

[11] Winfried Lohmiller and Jean-Jacques E Slotine. On contraction analysis for non-linear systems. Automatica, 34(6):683-696, 1998.

[12] Tad McGeer. Passive dynamic walking. The International Journal of Robotics Research, 9(2):62-82, 1990.

[13] Bastian Schürmann and Matthias Althoff. Guaranteeing constraints of disturbed nonlinear systems using setbased optimal control in generator space. IFAC-PapersOnLine, 50(1):11515 - 11522, 2017. 20th IFAC World Congress.

[14] Bastian Schürmann and Matthias Althoff. Optimal control of sets of solutions to formally guarantee constraints of disturbed linear systems. In 2017 American Control Conference, ACC 2017, Seattle, WA, USA, May 24-26, 2017, pages 2522-2529, 2017.

[15] Sho Shirasaka, Wataru Kurebayashi, and Hiroya Nakao. Phase reduction theory for hybrid nonlinear oscillators. Physical Review E, 95, 12017. 